\title{
Principles for Designing Activity-based Personalized eLearning
}

\author{
Owen Conlan, Ian O'Keeffe, Aoife Brady and Vincent Wade \\ Knowledge and Data Engineering Group, Dept. of Computer Science, \\ Trinity College Dublin, Ireland \\ \{Owen.Conlan,Ian.OKeeffe,Aoife.Brady,Vincent.Wade\}@cs.tcd.ie
}

\begin{abstract}
Activity-based Personalized eLearning involves delivering to a learner a learning experience that is tailored to their individual needs. Traditionally, these needs have been met through the personalized selection of content that fits the learner's preferences. However, by coupling this approach with the possibilities of interactive services a more engaging form of personalized education may be realized. This paper describes the design principles for producing activity-based personalized distance learning offerings.
\end{abstract}

\section{Introduction}

The research domain of personalized eLearning [1] offers a dynamic approach to personalization. Researchers in this domain attempt to create systems that dynamically personalize the educational experience to the evolving needs of the learner. These personalizations, however, tend to focus on the tailoring of content [2]. Again the potential of internet-based distance learning is not being fully realized - the educational experience offered needs to offer not only tailored content, but also tailored activities which are educational services that engage the learner through interaction.

Activity-based personalized distance learning offers learners unprecedented levels of engagement because the whole educational experience is tailored to their needs. For example, a personalized activity may involve using a virtual role play service where the responses to the learner are based on pre-existing knowledge about them. Moreover, the content offered to the learner may be contextualized to their current activity, thus delivering a holistic and personalized experience.

In order to achieve this vision the domains of personalized eLearning and service composition need to be combined to achieve the levels of integration required to deliver a holistic experience to learners. This paper describes how the multi-model approach [3] is being combined with the areas of Personalized Learning Content and Adaptive Web Service Composition to deliver a truly empowering distance learning solution.

\section{Background}

This section provides an introduction to the research areas of Personalized Learning and Adaptive Web Service Composition towards understanding the possibilities offered by these domains in achieving activity-based personalized eLearning.

\subsection{Personalized Learning Content}

Adaptive Hypermedia is the combination of various research efforts in the areas of user-modeling, adaptive systems and hypermedia. Brusilovsky [1] defines adaptive hypermedia as "all hypertext and hypermedia systems which reflect some features of the user in the user model and apply this model to adapt various visible aspects of the system to the user." In these systems, learning experiences are personalized to the needs of the individual learner based on the systems knowledge of the learner, for example their prior knowledge. Personalization is achieved through the application of techniques such as adaptive presentation as well as adaptive content selection and sequencing, known as adaptive navigation. Adaptation is facilitated by the modeling of the user, which the AHS uses to capture the learner's knowledge and preferences.

The Adaptive Personalized eLearning Service (APeLS) [3] implements a multi-model approach to providing an adaptive personalized course. This approach involves the runtime reconciliation of a set of metadata models (learner model, content model and narrative model) by an adaptive engine to produce the appropriate adaptation effects across the content.

KnowledgeTree [4] is a distributed architecture for adaptive eLearning which is based on the reuse of intelligent learning activities. It looks at the componentbased development of adaptive systems as well as reusability at the teacher level. The main services provided by KnowledgeTree are activity servers, valueadding servers, learning portals and student model servers.

A common feature of the systems discussed is that they all adopt a service orientated design approach, which facilitates all of the features of an adaptive eLearning system. The trend towards the use of services in this way 
is predominantly driven by the reusability of such services as well as its ability to support interoperability between different systems.

\subsection{Adaptive Web Service Composition}

Two common approaches to the composition of web services exist. The first is the use of workflow based techniques, relying on the manual selection and combination of services using languages such as the Web Service Business Process Execution Language (WSBPEL). This approach involves a 'developer' selecting and sequencing services from a set of known services allowing the desired goal to be met through composition. However, this technique only supports personalization to the extent that the composed services can provide through appropriate parameterization.

The second approach utilizes AI planning techniques [5] to automate the selection and sequencing process through analysis of the functional properties of the available services. Within the AI Planning domain there are various approaches that can be used to carry out the composition, these include the use of: situation calculus, planning graphs and hierarchical task networks [6].

The composition of web services can also be based on web services that are described using OWL-S. This is a logical step as OWL-S describes the same properties that AI planners use in the composition process.

\section{Principles of Personalized, Activity-based Learning}

Learners learn best when the learning experience offered to them meets their needs and stimulates their preferred modes of learning [7]. The approach discussed in this paper goes beyond the simple tailoring of the sequences of content to the personalization of the complete learning experience. As a result, the learner is presented with activities that have been personalized in order to meet their needs. This approach to learning empowers the learner using deeper levels of engagement. From a constructivist perspective [8], the learner becomes an active participant in the learning process, rather than solely a recipient. The challenge in presenting the learner with such opportunities lies in combining services, and offering activities and content that are educationally seamless. For example, there should not be a dramatic juxtaposition between participating in a learning activity and accessing personalized learning content. They should be strategically linked so that the overall experience of the learner is holistically consistent, ensuring that the educational experience is both effective and empowering.

The selection and sequencing of application-like components, which may be combined into activities, needs to be more precise than the sequencing content. If poor choices are made in content sequencing, the 'technical execution' of the overall course offering will not be adversely affected. However, mismatching content can result in an increase in the cognitive load placed on the learner as they try to understand the information presented.

An additional challenge in sequencing services is that of maintaining a cohesive workflow between these services. It is therefore important that the strategy guiding the composition process embodies a pedagogical approach which can create an educationally sound experience. At the same time there should be sufficient flexibility in the strategy, and in the services it combines, to cater for the personal and contextual preferences of the learner. The application of the multi-model approach to the composition of services may form the basis of the necessary relationship between services and strategies.

Rather than presenting a modular design which facilitates ease of reuse, most personalized eLearning systems, and specifically Intelligent Tutoring Systems (ITS), are monolithic systems which interweave intelligence with the content they are presenting. This lack of separation exists at both a system and content level. The multi-model, metadata driven approach describes a number of key principles for developing adaptive and personalized systems. These principles strive to make such systems easier to develop, reuse and repurpose.

Originally the multi-model approach described the theoretical models and associated implementation of a generic and extensible adaptive metadata driven engine that composed, at runtime, tailored educational experiences across a single educational content base. This restriction has evolved, with the advent of service oriented architectures, to encompass separate and diverse content and activity sources. The fundamental principles, however, remain the same. These are:

- Model and Narrative Separation

- Metadata Representing Models

- Abstraction through Candidacy and Candidate Selection

- Runtime Reconciliation and Multiple Adaptive Services

Model and Narrative Separation is the most fundamental element of the multi-model approach. This consists of the separation of the constituent information models, upon which the adaptivity is based, into discrete and separate models. This separation also applies to the adaptive sequencing and adaptive composition logic, referred to as Narrative in this work. For example, a simple personalized eLearning system may have a learner model, domain model and narrative model, where the narrative is responsible for selecting the appropriate content for the learner. The value of this approach is that the models can evolve and grow independently as well as facilitating the addition of other models to the system. For 
instance, if we wished to add a context model, representing the computing device and environment, to the personalized eLearning system. In this case, the only impact would be on the narrative model, which must now reconcile this additional evidence in the personalization process.

Obviously, it is not possible to add an actual learner to a database, therefore a meta-representation is the most we can achieve. The multi-model, metadata driven approach prescribes that all models are described using metadata. This is another fundamental element as it is through this metadata that reconciliation can occur. For example, if there are three pieces of content to choose from it is only through processing their metadata in accordance with the narrative and with regard to other models that a selection can be made.

By applying the multi-model approach it is also possible to make decisions based on abstract concepts. This abstraction is made possible through the use of candidacy and candidate selection. Candidacy allows an adaptive system to make only the decisions it is capable of making and deferring certain decisions to the learner or to another system. Alternatively, the decision can be deferred until the appropriate information becomes available. Simply put, candidacy enables similar models to be grouped. For example, if there are three pieces of content that teach Pythagoras' Theorem, each utilizing a different learning style, then they may be grouped into a single candidate group. The strength of this approach is that the decision to choose one piece of content, service or narrative over another can be deferred until the learner needs to access it. This leads to just-in-time personalization allowing the system to utilize the most upto-date metadata when making a selection. Furthermore, the selection may be deferred to another personalization service allowing a separation of logic and responsibility. As narratives are also considered to be models there may also exist multiple candidate narratives, referred to as subnarratives, which represent alternative logics, thus enabling different elements of strategy to be selected at runtime.

The significant difference between adaptively sequencing content and sequencing services is the need to manage data flow between services. This data takes the form of parameters for launching the services or manipulation of data models upon which to make decisions.

Through candidacy it also becomes possible to defer the decision to another service which may be more suited to making that decision. In this way a personalized service does not need to grow into a monolithic system attempting to resolve all issues involved in personalization, rather it can specialize and leave decisions outside of its expertise to other services. Therefore, it is possible to envisage a suite of adaptive services cooperating together to produce a multiply adapted outcome.

\section{Conclusions}

This paper has described the principles behind the combination of personalized content and services may be used to create activity-based personalized distance learning offerings. Through combining the methodologies seen in the domains of service composition and personalized learning a service-oriented approach to personalized learning activities may be realized. The principles espoused by the multi-model, metadata driven approach have been shown as appropriate guidelines for achieving successful personalizations that engage learners in activity-based distance learning opportunities. The work described in this paper is currently being applied in the European Commission FP6 IST project iClass to provide rich personalised learning experiences to $\mathrm{K} 12$ school children.

\section{References}

[1] P. Brusilovsky: Methods and techniques of adaptive hypermedia. In journal User Modeling and User-Adapted Interaction, Volume 6, pp. 87-129, Kluwer Academic Publishers, 1996.

[2] P. De Bra and L. Calvi: AHA: a Generic Adaptive Hypermedia System. In Proceedings of the 2nd Workshop on Adaptive Hypertext and Hypermedia, pp. 5-12, Pittsburgh, 1998. [3] O. Conlan, V. Wade, C. Bruen, M. Gargan: Multi-Model, Metadata Driven Approach to Adaptive Hypermedia Services for Personalized eLearning. In Proceedings of the Second International Conference on Adaptive Hypermedia and Adaptive Web-Based Systems, Lecture Notes in Computer Science, Springer Berlin, Volume 2347, pp. 100-111, 2002.

[4] P. Brusilovsky. KnowledgeTree: A distributed architecture for adaptive e-learning. In Proceedings of the Thirteenth International World Wide Web Conference, Alternate Track Papers \& Posters, Association for Computing Machinery, Inc. (ACM), pp. 104-113. New York, May 2004.

[5] M. Fox and D. Long: The Third International Planning Competition: Temporal and Metric Planning. In Proceedings of the Sixth International Conference on Artificial Intelligence Planning and Scheduling, AAAI Press, pp. 333-335. Toulouse, France, April 2002.

[6] J. Rao, X. Su: A survey of automated web service composition methods. In Proceedings of the First International Workshop on Semantic Web Services and Web Process Composition SWSWPC, Springer-Verlag, pp. 43. San Diego, California, USA, July, 2004.

[7] O. Conlan and V. Wade: Evaluation of APeLS - An Adaptive eLearning Service based on the Multi-model, Metadata-driven Approach. Third International Conference on Adaptive Hypermedia and Adaptive Web-Based Systems (AH2004), edited by De Bra, P., Nejdl, W., Springer, pp291 - 295, 2004.

[8] M. W. Caprio. Easing into constructivism, connecting meaningful learning with student experience. Journal of College Science Teaching, 23 (4), pp210-212, 1994. 